\title{
Comparison Study on SU-8 and Its Composites
}

\author{
Bhaskar Radhe Shyam \\ Department of Mechanical Engineering, JP Engineering College, Rajpura, Meerut, India
}

\section{Email address:}

02cim04@gmail.com

\section{To cite this article:}

Bhaskar Radhe Shyam. Comparison Study on SU-8 and Its Composites. American Journal of Aerospace Engineering. Vol. 6, No. 2, 2019, pp. 33-38. doi: 10.11648/j.ajae.20190602.11

Received: July 20, 2019; Accepted: December 25, 2019; Published: January 8, 2020

\begin{abstract}
In this paper, SU-8 is an industrially useful photo resist polymer for micro-fabrication because of its unique UV sensitive curing property. It is also used as a structural material for micro-machines such as micro-electro mechanical systems (MEMS). However, it has poor tribological and mechanical properties which make SU-8 inferior to Si, the mainstay MEMS material today. In this paper, we report the fabrication of SU-8 nanocomposites which are self-lubricating and have better mechanical properties. The liquid lubricant i.e., perfluoropolyether (PFPE) and nanoparticles such as $\mathrm{SiO}_{2}$, CNTs, and graphite were added into SU-8 for this purpose. These self-lubricating SU-8 + PFPE and SU-8 + PFPE + nanoparticle composites have shown a reduction in the initial coefficient of friction, increased wear life and the mechanical properties such as the elastic modulus and the hardness have increased. We have used perfluoropolyether (PFPE) as in-situ lubricant filler to SU-8. This composite material has shown highly superior friction and wear performance over pristine SU-8 and any such alternative material for this application. We have shown that the mechanical property can also be enhanced by this method if we utilize nano-particles for further reinforcement. This self-lubricating SU-8+PFPE composite can be used for the fabrication of MEMS applications requiring no external lubrication, and also these composites can find applications in many tribological components of traditional machines. The thickness of SU-8 and its composites coating is fabricated in the range $\sim 100-105 \pm \mathrm{m}$. Further, SU-8 and its composites are characterized by a 3D optical profilometer, atomic force microscopy, scanning electron microscopy, a thermal gravimetric analyzer, a goniometer, a hardness tester, and an optical microscope. Under a tribology test performed at different normal loads of 2,4 , and $6 \mathrm{~N}$ and at a constant sliding speed of $0.28 \mathrm{~m} / \mathrm{s}$. The SU-8 composite reinforced with $10 \mathrm{wt} . \% \mathrm{~h}-\mathrm{BN}$ and $20 \mathrm{wt} . \%$ PFPE demonstrated the best thermo-mechanical and tribological properties with a nano-textured surface of high hydrophobicity.
\end{abstract}

Keywords: Su-8, Boron Nitride, Perfluoropolyether (PFPE), Self Lubrication, MEMS, Base Oil (SN-150)

\section{Introduction}

SU-8 is a commonly used epoxy-based negative photoresist. Negative refers to a photoresist whereby the parts exposed to UV become cross-linked, while the remainder of the film remains soluble and can be washed away during development. Microsystems such as MEMS (micro electro mechanical systems) are small scale devices (from few $\mu \mathrm{m}$ to several hundred $\mu \mathrm{m}$ ), designed to perform variety of operations in various industries including automotive, bio-engineering, bio-medical and military etc. As the surface area to volume ratio gets larger, the surface forces become highly detrimental to operation of these devices. These forces, for small devices, are capable of stopping the operation of machine against its inertial forces [1]. SU-8 consists of three basic components: (a) an EPON ${ }^{\mathrm{TM}} \mathrm{SU}-8$ epoxy resin; (b) a solvent such as gamma-butyrolactone (GBL) and (c) a photoacid generator such as triaryl sulfonium salts. Each SU-8 molecule consists of an average of 8 epoxy groups (and hence the name SU-8). In order to improve the tribology of SU-8 through self-lubrication, we have developed bulk SU-8 composites by using the liquid lubricants such as PFPE lubricant and nano-fillers such as $\mathrm{SiO}_{2}, \mathrm{CNTs}$ and graphite as filler materials. These SU-8 composites have yielded much improved tribological properties when compared to the pristine SU-8 [2, 3].

A photo resist is a light-sensitive material used in several processes, such as photolithography and photoengraving, to form a patterned coating on a surface. This process is crucial in the electronic industry. 


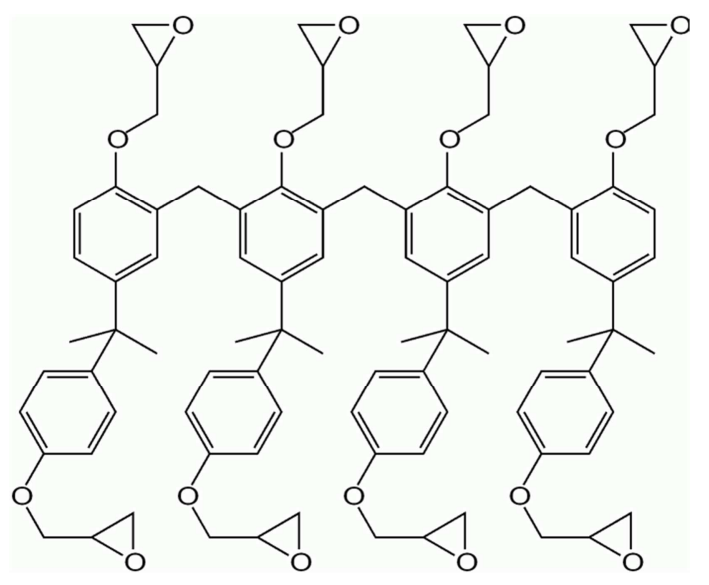

Figure 1. Structure of SU-8 molecule.

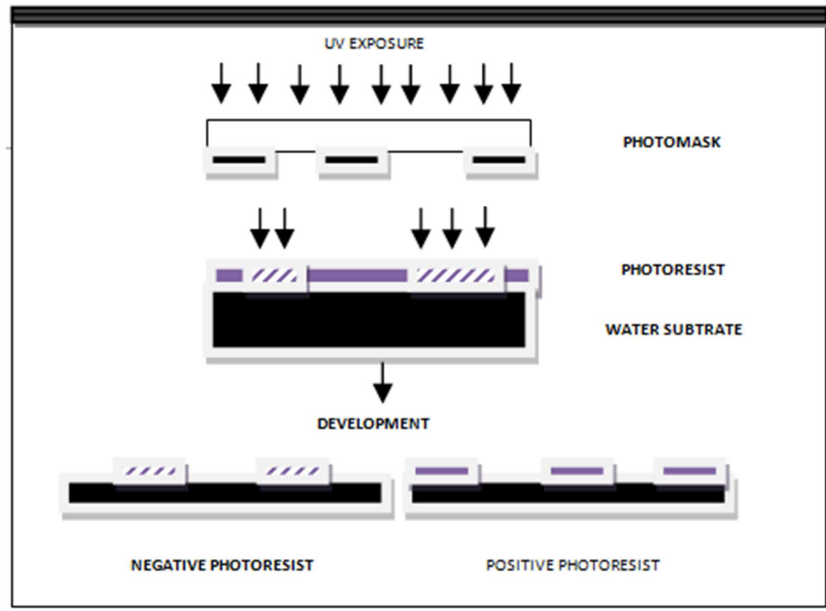

Figure 2. Negative and positive photo resist.

Surface treatments and coatings have provided tremendous achievements in micro-electro-mechanical system (MEMS) industries from metallurgy to processing [3]. Composite deposits can be coated on steel, light metals, ceramics, plastics, and other materials to enhance the surface properties, such as mechanical, tribological, corrosive, and thermal [4].
For all polymeric materials, SU-8 is a promising material for next generation 3D fabrication. It can also be used as a coating material in different applications, such as bearing steel [5]. Currently, silicon wafers are used for the fabrication of MEMS devices; however, they have several disadvantages, such as a low thermal stability, difficulty in 3D fabrication, and non-biocompatibility. These disadvantages can be diminished by introducing a polymer, such as SU-8 [6].

Therefore, researchers have proposed to mix liquid lubricant fillers with or without solid fillers in SU-8. Liquid lubricant fillers, such as PFPE, MAC, and SN 150 have been mixed with solid fillers, such as CNT and graphite, in an SU8 matrix, and a tribology test was performed on a ball-ondisk machine. The wear resistance property and wear life were improved by $\sim 105$ times. Moreover, the initial and steady-state coefficients of friction were reduced $[7,8]$.

\section{Comparison Study}

\subsection{AFM Images}

AFM images of freshly spin-coated Pristine SU-8 and SU8+PFPE composite surfaces before and after washing [2, 9]. AFM images of freshly spin-coated surfaces of pristine SU-8 and SU-8+PFPE composite, before and after washing. The pristine SU-8 shows plain surface without any feature.

\subsection{Water Contact Angles}

The pristine $\mathrm{SU}-8$ and $\mathrm{SU}-8$ nanocomposites were characterized for their static water contact angles (WCAs) using VCA Optima Contact Angle System (AST Products Inc, USA). A water droplet of 0.511 was used for contact angle measurements. At least five replicate measurements, for three different samples, were carried out and an average value is reported in the paper. The variation in WCAs at various locations of a sample was within \pm 3 . The measurement error was within \pm 1 . The WCAs were also measured inside the wear tracks after tribological tests.

Table 1. WCA measurement for all SU-8 based nano composites with and without PFPE over fresh (pristine surface of composites before experiment) and worn surfaces (wear track or the spot where the sliding test was conducted).

\begin{tabular}{lll}
\hline \multirow{2}{*}{ Sample description } & WCA measurement in degrees & \\
\cline { 2 - 3 } & On fresh surface & On worn surface (wear track) \\
\hline Bare $\mathrm{SU}-8$ & 84 & 54 \\
$\mathrm{SU}-8+\mathrm{CNTs}$ & 89 & 60 \\
$\mathrm{SU}-8+\mathrm{SiO}_{2}$ & 85 & 54 \\
$\mathrm{SU}-8+$ graphite & 79 & 50 \\
$\mathrm{SU}-8+\mathrm{PFPE}$ & 98 & 94 \\
$\mathrm{SU}-8+\mathrm{PFPE}+\mathrm{CNTs}$ & 91 & 120 \\
$\mathrm{SU}-8+\mathrm{PFPE}+\mathrm{SiO}_{2}$ & 106 & 105 \\
$\mathrm{SU}-8+\mathrm{PFPE}+\mathrm{graphite}$ & 91 & 97 \\
\hline
\end{tabular}

The data reported here for bare SU-8 and SU-8+NP composites without PFPE were recorded after $10^{\wedge} 4$ cycles and for SU-8+NP composites with PFPE were recorded after $10^{\wedge} 6$ cycles, respectively
Steady-state values are not mentioned for specimens that did not contain PFPE as these specimens failed right in the first sliding cycle according to the wear-failure criteria followed in this study. 


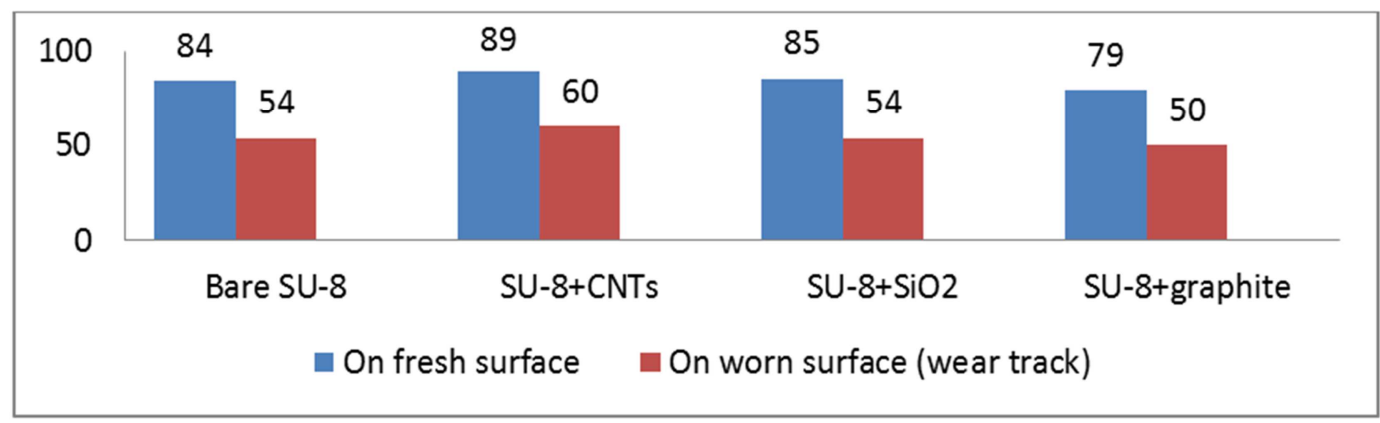

(a)

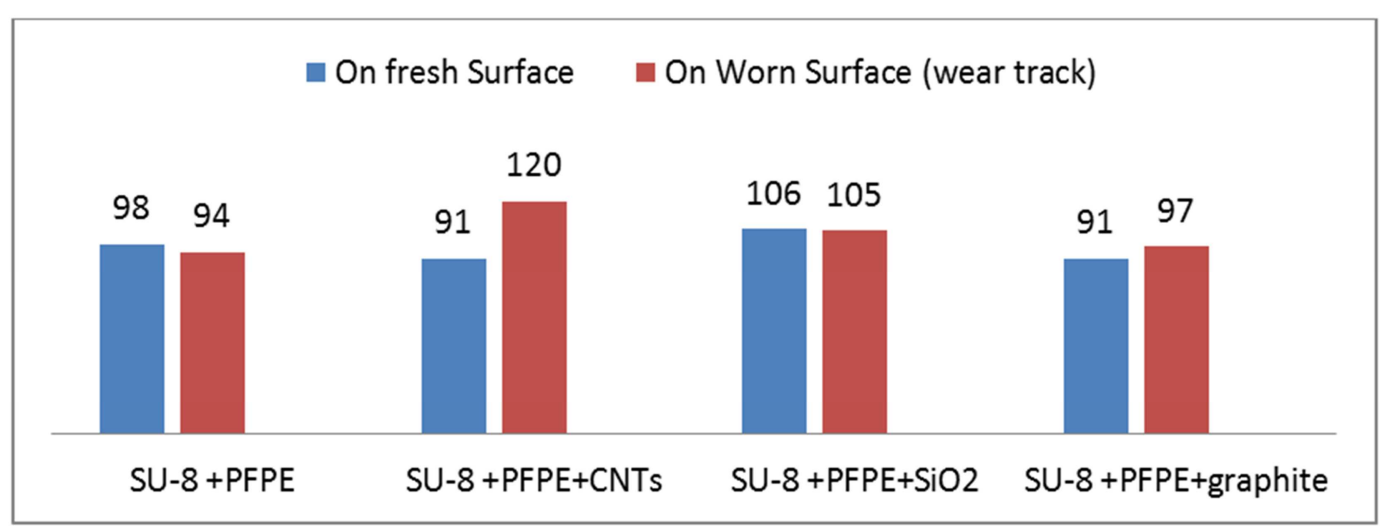

(b)

Figure 3. WCA values for pristine SU-8 and SU-8 based composites over fresh (pristine surface of composites before experiment) and worn surfaces (wear track). (a): Non-PFPE combinations at 104 cycles (composites did not contain PFPE). (b): PFPE combinations at 106 cycles.

There is $100 \%$ increase in WCA values from non-PFPE combinations to PFPE combinations in worn surface condition. It is clear that PFPE is responsible for this enhanced hydrophobic behavior.

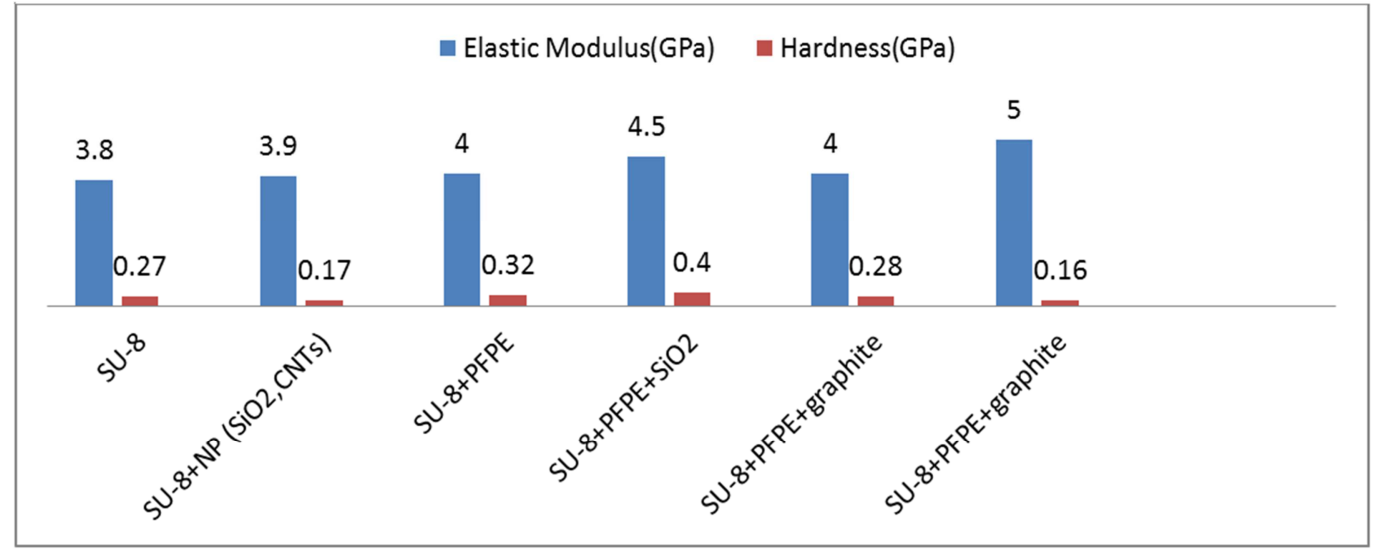

Figure 4. Elastic Modulus (E) and Hardness (H) values of pristine SU-8 and SU-8 composites from nano-indentation characterization.

\subsection{X-ray Photoelectron Spectroscopy (XPS): \\ Characterization}

XPS characterization of pristine SU-8 and SU-8 + PFPE composite was done using VG ESCA Lab-220i XL XPS. Mg Ka X-ray was employed for the analysis of one spot on each sample with photoelectron take-off angle of $90^{\circ}$. The analysis area was $4 \mathrm{~mm} \mathrm{X} 4 \mathrm{~mm}$, whereas the maximum analysis depth lies in the range of $\sim 4-8 \mathrm{~nm}$. XPS characterization was also done inside the wear track after tribological tests for SU-8 + PFPE samples using the Thermo Fisher Scientific Thetaprobe
XPS. Monochromatic Al Ka X-ray was employed for the analysis of one spot on each sample with photoelectron takeoff angle of 50 (with respect to surface plane normal). The analysis area was $100 \mu \mathrm{m}$ X $100 \mu \mathrm{m}$. The maximum analysis depth lay in the range of $\sim 4-8 \mathrm{~nm}$. A specially designed electron flood gun with a few eV Ar+ ion was used for the charge compensation. Electron beam and ion beam were focused and steered toward the analysis position. The voltage of the X-ray beam is $15 \mathrm{kV}$. The pass energies for survey scan and high resolution scans were 200 and $40 \mathrm{eV}$ respectively. 


\subsection{Coefficient of Friction Versus Number of Cycles}
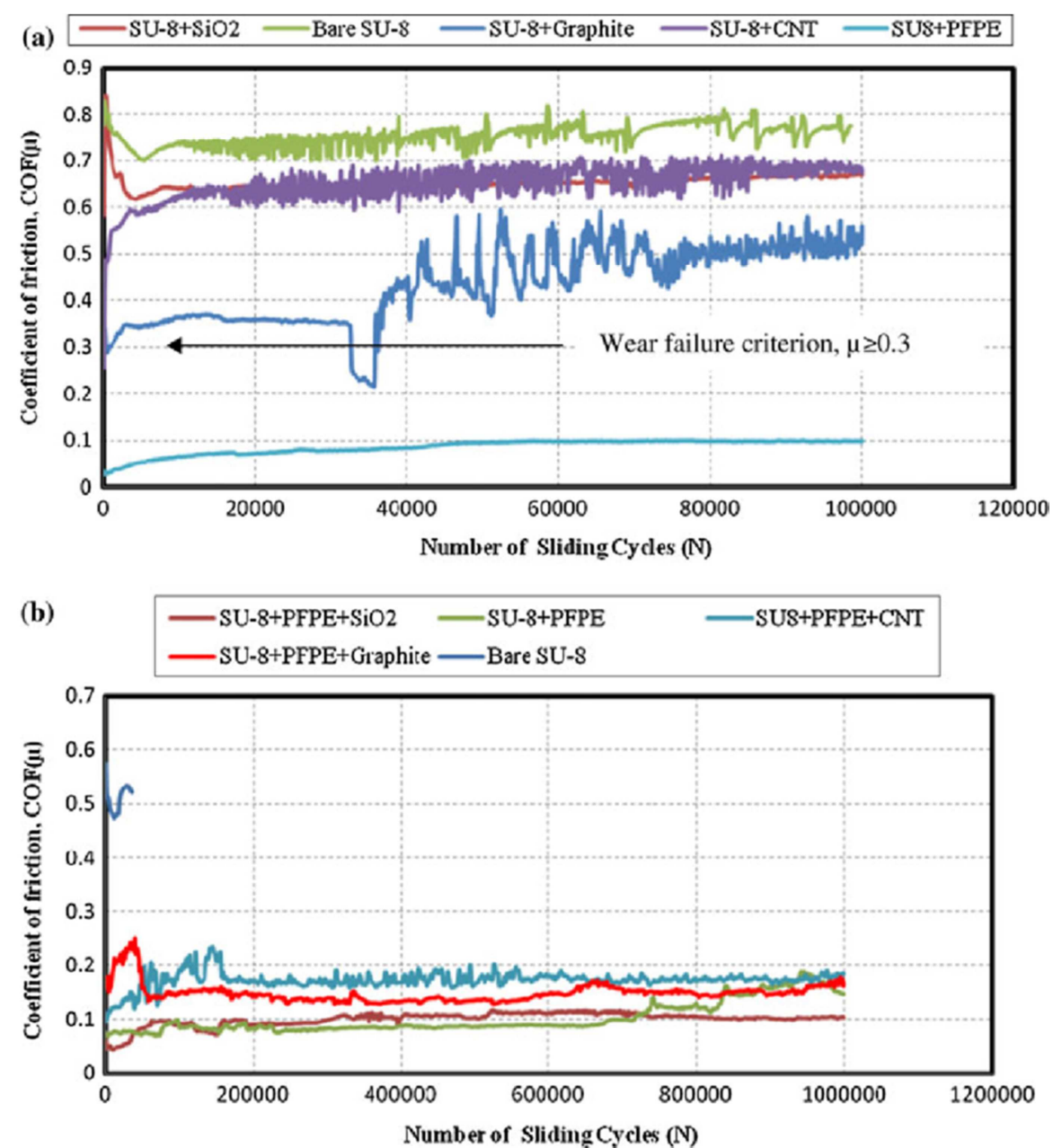

Figure 5. A Coefficient of friction versus number of cycles data for $\mathrm{SU}-8, \mathrm{SU}-8+\mathrm{PFPE}, \mathrm{SU}-8+\mathrm{SiO}_{2}, \mathrm{SU}-8+\mathrm{CNTS}$, and $\mathrm{SU}-8+$ graphite nanocomposites obtained from the ball-on-disk sliding tests against $4 \mathrm{~mm}$ diameter $\mathrm{Si}_{3} \mathrm{~N}_{4}$ ball at a normal load of $30 \mathrm{~g}$ and a sliding rotation of $200 \mathrm{rpm}$. $b$ Coefficient of friction versus number of cycles data for $S U-8, S U-8+P F P E, S U-8+P F P E+S i O_{2}, S U-8+P F P E+C N T$, and SU-8 + PFPE + graphite obtained from the ball-on-disk sliding tests against $4 \mathrm{~mm}$ diameter $\mathrm{Si}_{3} \mathrm{~N}_{4}$ ball at a normal load of $300 \mathrm{~g}$ and a sliding rotation of 2,000 rpm. The tests were stopped at one million cycles because of the long test duration as the samples had not failed.

\subsection{Optical Micrographs of Worn Surfaces}

In the current work, the improved tribological behavior of SU-8 nanocomposites was also ascertained at very low loads of 10 and $5 \mathrm{~g}$ and at high speed of 3,000 rpm (data are not included) times. The presence of PFPE film at the interface due to in situ lubricant supply is the main reason for superior tribological performance whereas the nanoparticles help in the mechanical property enhancement. These SU-8 nanocomposites can be used as self-lubricating structural materials for MEMS requiring no external lubrication. Apart from this, the SU-8 nanocomposites can also find application as a lubricious and wear resistant coating for several tribological components made from different materials. Examples are journal bearings, raceways of a ball bearing, gears, medical equipments and tools, bio-devices, precision positioning stages, electronics components such as those inside cameras and printers, plastic bearings, etc.

Table 2. Elastic modulus (GPa) and hardness (GPa) of SU-8 and SU-8 nanocomposites obtained through nanoindentation tests using MTS Nano Indenter XP with a CSM technique.

\begin{tabular}{lll}
\hline Nanocomposite description & Elastic modulus (GPa) & Hardness (GPa) \\
\hline $\mathrm{SU}-8$ & 3.8 & 0.27 \\
$\mathrm{SU}-8+\mathrm{NP}\left(\mathrm{SiO}_{2}, \mathrm{CNTs}\right)$ & 3.9 & 0.17 \\
$\mathrm{SU}-8+\mathrm{PFPE}$ & 4.0 & 0.32 \\
$\mathrm{SU}-8+\mathrm{PFPE}+\mathrm{SiO}_{2}$ & 4.5 & 0.40 \\
$\mathrm{SU}-8+\mathrm{PFPE}+\mathrm{CNTs}$ & 4.0 & 0.28 \\
$\mathrm{SU}-8+\mathrm{PFPE}+$ graphite & 5.0 & 0.016 \\
\hline
\end{tabular}




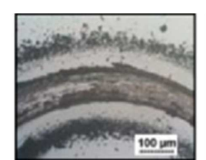

(a)

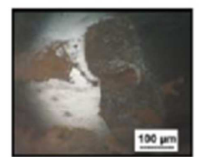

(g)

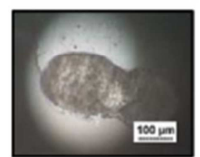

(m)

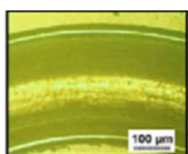

(b)

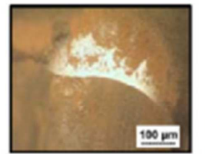

(h)

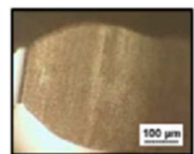

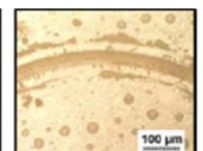

(c)

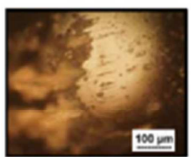

(i)

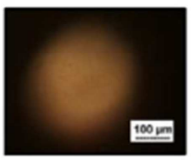

(o)

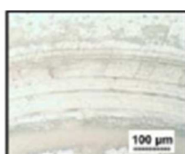

(d)

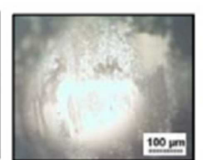

(j)

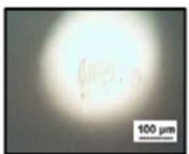

(p)

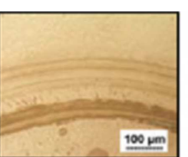

(e)

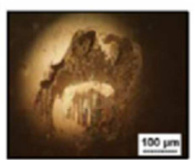

(k)

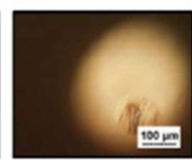

(q)

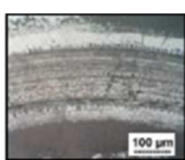

(f)

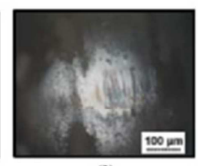

(I)

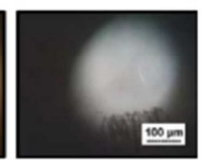

(r)

Figure 6. Optical micrographs of worn surfaces: a Bare SU-8 (at 10,000 cycles), b SU-8 + nanoparticles (SiO 2 , CNT) (at 10,000 cycles), c SU-8 + PFPE (at one million cycles), $d$ SU-8 + PFPE + CNTs (at one million cycles), e SU-8 + PFPE + SiO 2 (at one million cycles), and f SU-8 + PFPE + graphite (at one million cycles). $g, h, i, j, k, l$ and $m, n, o, p, q, r$ are optical micrographs of the counter face balls surface after sliding tests and micrographs of the tested counter face balls after cleaning with acetone corresponding to the worn surfaces shown in $a, b, c, d$, e, and $f$, respectively. The length of the scale bar is 100 Im in all images.

\section{Conclusions}

In this comparison study, adding the fillers, textures were generated on the surface, which changed the surface from hydrophilic to hydrophobic. The developed self-lubricating SU8 composites with PFPE are much more hydrophobic than SU-8 and SU-8+NP composites in fresh and worn surface conditions. The self-lubricating SU-8 composites reduced initial coefficient of friction of SU-8 by $\sim 6-9$ times and increased wear life by $>105$ times. The elastic modulus $(\mathrm{E})$ and hardness $(\mathrm{H})$ were also increased by $\sim 1.4$ times. However, after adding lubricant fillers with h-BN, the initial and steady-state coefficients of friction were reduced by $\sim 8$ times and $\sim 9$ times, respectively. Moreover, the composite coating improved the mechanical and surface properties. Therefore, the composite coating could be useful for bearing applications and MEMS fabrication. Apart from this, the SU-8 nanocomposite can also find application as a lubricious and wear resistant coating for several tribological components made from different materials. Examples are journal bearings, raceways of a ball bearing, gears, medical equipments and tools, biodevices, precision positioning stages, electronics components such as those inside cameras and printers, plastic bearings, etc.

\section{References}

[1] Kim, S. H., Asay, D. B., and Dugger, M. T., "Nanotribology and MEMS", NanoToday 2, 2007, 22-29.

[2] Sujeet K. Sinha, Prabakaran S., Duong H. M., "SU-8 Composites for Micro-systems applications" Journal of Physics D: applied, 2013, 1-4.

[3] Prabakaran S, Satyanarayana N and Sinha SK., "Selflubricating SU-8 nano-composites for microelectromechanical systems applications", Tribology Letters 49 (1), 2013, 169178 .
[4] Anand Singh R., Jitendra S. Katiyar, Vinay K Patel, "Thermomechanical and tribological properties of SU-8/h-BN composites with SN 150/ perfluoropolyether (PFPE) filler" Springer 2019, 1-13.

[5] Gelorme, J. D., Cox, R. J., and Gutierrez, S. A. R., "Photoresist composition and printed circuit Boards and packages made herewith", US Patent 4, 882, 245, Date of Patent: Nov. 21, 1989.

[6] Abgrall, P., Conedera, V., Camon, H., Gue, A-M., and Nguyen, N. T., "SU-8 as a structural material for labs-on-chips and microelectromechanical systems", Electrophoresis 28, 2007, $4539-4551$.

[7] Saravanan P, Satyanarayana N, Sinha S K. Wear durability study on self-lubricating SU-8 composites with perfluoropolyther, multiply-alkylated cyclopentane and base oil as the fillers. Tribol Int 64: 103-115 (2013).

[8] Saravanan P, Satyanarayana N, Siong P C, Duong H M, Sinha S K. Tribology of self-lubricating SU-8+PFPE composite based Lub-tape. Procedia Eng 68: 497-504 (2013).

[9] Prabakaran Saravanan, Nalam Satyanarayana and S. K. Sinha, "Wear Durability Study on Self-lubricating SU-8 composites with perfluoropolyther, multiply-alkylated cyclopentane and base oil as the fillers", Tribology International, 64 (2013) 103-115.

[10] Jiguet, S., Judelewicz, M., Mischler, S., Bertch, A., and Renaud, P., "Effect of filler behavior on nanocomposite SU8 photoresist for moving micro-parts", Microelectronic Engineering 83, 2006, 1273-1276.

[11] Marinis, T. F.: The future of microelectromechanical systems (MEMS). Strain 45, 208-220 (2009).

[12] Kim, S. H., Asay, D. B., Dugger, M. T.: Nanotribology and MEMS. NanoToday 2, 22-29 (2007).

[13] de Boer, M. P., Mayer, T. M.: Tribology of MEMS. MRS Bull. 4, 302-304 (2001).

[14] Lee, K. K., Bhushan, B., Hansford, D.: Nanotribological characterization of fluoropolymer thin films for biomedical micro/ nanoelectromechanical system applications. J. Vac. Sci. Technol. A 23, 804-810 (2005). 
[15] Gelorme, J. D., Cox, R. J., Gutierrez, S. A. R.: Photoresist composition and printed circuit boards and packages made herewith. US Patent 4, 882, 245, 21 Nov 1989 6. Abgrall, P., Conedera, V., Camon, H., Gue, A. -M., Nguyen, N. T.: SU-8 as a structural material for labs-on-chips and microelectromechanical systems. Electrophoresis 28, 45394551 (2007).

[16] Bertsch A, Renaud P. Special issue: 15 years of SU8 as MEMS material. Micromachines 6 (6): 790-792 (2015).

[17] Steinhäuser S, Wielage B. Composite coatings: Manufacture, properties, and applications. Surf Eng 13 (4): 289-294 (1997)/
[18] Rathaur A S, Katiyar J K, Patel V K, Bhaumik S, Sharma A K. A comparative study of tribological and mechanical properties of composite polymer coatings on bearing steel. Int J Surf Sci Eng 12 (5-6): 379-401 (2018).

[19] Lau K H, Giridhar A, Harikrishnan S, Satyanarayana N, Sinha S K. Releasing high aspect ratio SU-8 microstructures using AZ photoresist as a sacrificial layer on metallized Si substrates. Microsyst Technol 19 (11): 1863-1871 (2013). 